\title{
Desingularization of Flexible-Joint Parallel Robots
}

\section{Mustafa Özdemir ${ }^{1, *}$ and Sıtkı Kemal İder²}

${ }^{1}$ Department of Mechanical Engineering, Faculty of Engineering, Marmara University, Göztepe Campus, 34722 Kadıköy, Istanbul, Turkey

E-mail: mustafa.ozdemir@marmara.edu.tr ORCID iD: (D) https://orcid.org/0000-0002-4981-9573

* Corresponding author

${ }^{2}$ Department of Mechanical Engineering, Faculty of Engineering, Çankaya University, 06790 Etimesgut, Ankara, Turkey

E-mail: kider@cankaya.edu.tr

ORCID iD: (D) https://orcid.org/0000-0001-5869-893X

Abstract: Parallel robots possess a characteristic type of singularities, called type II or drive singularities, inside their workspace. In the neighborhood of these singularities, the inverse dynamics solution grows unboundedly and the robot becomes uncontrollable. There is growing literature on methods that enable parallel robots to pass through drive singularities. Most of this literature relies on dynamic models that presume rigid joints. However, the flexibility of the drive train elements should also be taken into account for high accuracy. In this paper, we propose a systematic trajectory planning method for enabling flexible-joint parallel robots to pass through drive singular configurations. Our method generates admissible polynomial trajectories of degree eleven. Four conditions are derived and incorporated into the method to prevent undesired back-and-forth motion of the endpoint. This ensures not only an efficient operation of the robot but also the avoidance of unintended multiple occurrences of the same singularity. The boundedness of the inverse dynamics solution is also guaranteed.

Keywords: flexible-joint parallel robot; drive train flexibility; drive singularity; singularity removal; trajectory planning

\section{Introduction}

Parallel robots are used in a wide variety of applications and promise many more. For this reason, numerous studies have been devoted to the design and improvement of various parallel robots. Some recent works in this regard are [1-4]. However, "type II singularities" [5] or "drive singularities" [6] constitute a 
challenging issue in the use of parallel robots. The inverse dynamics solution is unbounded near these singularities, which results in saturation of the actuators and loss of control over the robot. Since such singularities lie generally in the interior of the workspace [5], a considerable amount of research has been conducted to develop trajectory planning methods for enabling parallel robots to pass through them with bounded actuator efforts. Ider [6] derived the "consistency conditions" in terms of the generalized accelerations as necessary conditions to be satisfied at the singular positions. Jui and Sun [7] introduced an approach that considers the bounds of the actuators. Briot and Arakelian [8] formulated an equivalent condition for consistency in terms of the forces and moments exerted on the moving platform. Özdemir [9] proved that although the consistency of the robot's dynamic equations at singular configurations is necessary, it is not sufficient to guarantee the boundedness of actuator efforts, and derived the additional necessary conditions in this respect by taking into account the time derivatives of the associated vanishing determinant at the instant of singularity. The conditions under which a planar 5R parallel robot experiences "high-order singularities" were derived in [10]. As remarked in [11], a trajectory planned to achieve the consistency of the dynamic model at the singularity may result in unintended back-and-forth motion of the endpoint. A method was proposed in [12] to prevent the possibility of such an undesirable motion.

All the above-mentioned studies on the trajectory planning of parallel robots are based on the assumption of nonredundant actuation. But when the effects of flexibility arising at the actuated joints due to the elasticity and structural damping of the drive train elements are significant, the robot should be treated as an underactuated system with doubled number of degrees of freedom [13, 14]. Indeed, the issues related to the dynamics, trajectory planning, and control of such a system are quite different from those of its counterpart with rigid drive trains. Spong [13] proposed to model the elasticity of the joint as a linear torsional spring. Structural damping can be taken into account by adding a linear torsional damper in parallel to the model [15]. Although there are several studies [16-18] on the control and optimization of flexible-joint parallel robots, the motion is confined to be singularity-free in these studies and the literature is very limited on their trajectory planning for passing through drive singularities. Considering the torsional elasticity of the actuated joints, it was suggested in [19] that the degree of the trajectory polynomial must be at least twelve to pass through a singularity.

In the present paper, a novel trajectory planning method is proposed for desingularization of flexible-joint parallel robots, by which an admissible trajectory can be systematically generated using an eleventh-degree polynomial, that is, using a polynomial of a lower degree than that required in the related literature [19]. This degree reduction from twelve to eleven is particularly important since it also enables to solve the problem of avoiding any undesired reverse motion of the endpoint. Such a back-and-forth motion is obviously undesirable from an efficiency perspective. However, more importantly, it may 
lead to some additional unintended occurrences of the same singularity, as shown for the first time in the present paper. Although a singularity is removed at the intended time of passing through its corresponding configuration, the dynamic equations will be, in general, inconsistent at the times of its unplanned additional occurrences, yielding an unrealizable trajectory. Hence, such occurrences should be avoided, and when an eleventh-degree polynomial is used, the avoidance of reverse motion is guaranteed by ensuring the nonnegativity of a second-degree time polynomial over the task duration. The boundedness of the inverse dynamics solutions is also guaranteed by taking into account the first-order time derivative of the associated vanishing determinant, in accordance with $[9,10]$.

\section{Dynamic Modelling of Flexible-Joint Parallel Robots}

Consider a rigid-link flexible-joint parallel robot with $n$ rigid degrees of freedom and $n$ motors at the actuated joints. The polar mass moment of inertia of the rotor of the $i^{\text {th }}$ motor $(i=1, \cdots, n)$ is represented by $J_{i}$. Suppose that the rotors have symmetric mass distributions about their rotation axes [13]. The gear ratio of the speed reducer of the $i^{\text {th }}$ motor is $R_{i}$. The angular displacement and torque of the output shaft of the speed reducer of the $i^{\text {th }}$ motor are denoted by $\theta_{i}$ and $\tau_{i}$, respectively. Define two $n \times 1$ vectors $\boldsymbol{\theta}$ and $\boldsymbol{\tau}$ as $\boldsymbol{\theta}=\left[\begin{array}{lll}\theta_{1} & \cdots & \theta_{n}\end{array}\right]^{\mathrm{T}}$ and $\boldsymbol{\tau}=\left[\begin{array}{lll}\tau_{1} & \cdots & \tau_{n}\end{array}\right]^{\mathrm{T}}$. Notice that $\tau_{i}, i=1, \cdots, n$, are the input torques of the robot.

The elasticity and structural damping of the coupling between the output shaft of the speed reducer of the $i^{\text {th }}$ motor and the corresponding driven link is modeled by a parallel arrangement of a linear torsional spring with a spring constant $k_{i}$ and a linear torsional damper with a damping coefficient $c_{i}$ [15]. Notice that there are $n$ additional degrees of freedom due to the flexibility at the actuated joints. Let $q_{i}^{\mathrm{a}}$ represent the angular displacement of the link that is coupled to the output shaft of the speed reducer of the $i^{\text {th }}$ motor, the angular deflection of this coupling being $\delta_{i}=q_{i}^{\mathrm{a}}-\theta_{i}$. Define the vector of actuated joint variables, $\mathbf{q}^{\mathrm{a}}$ as $\mathbf{q}^{\mathrm{a}}=\left[\begin{array}{lll}q_{1}^{\mathrm{a}} & \cdots & q_{n}^{\mathrm{a}}\end{array}\right]^{\mathrm{T}}$.

It is convenient to study first the dynamics of the rigid-joint counterpart of the robot, where each motor (including its rotor and gears) is treated as a point mass fixed to the link on which the stator is attached [13, 15]. This closed-loop mechanism can be converted into an open-tree system by virtually cutting a sufficient number of unactuated joints. Let this open system have $m$ degrees of 
freedom, with a vector of joint variables $\mathbf{q}=\left[\begin{array}{lll}q_{1} & \cdots & q_{m}\end{array}\right]^{\mathrm{T}}$. Without loss of generality, the elements of the vector $\mathbf{q}$ are assumed to be ordered such that

$\mathbf{q}=\left[\begin{array}{l}\mathbf{q}^{\mathrm{a}} \\ \mathbf{q}^{\mathrm{u}}\end{array}\right]$

where $\mathbf{q}^{\mathrm{u}}=\left[\begin{array}{lll}q_{1}^{\mathrm{u}} & \cdots & q_{m-n}^{\mathrm{u}}\end{array}\right]^{\mathrm{T}}$ is the vector of unactuated joint variables. If the input torques are the only applied nonconservative forces, the equations of motion of the open system can be written using the Lagrangian method as

$$
\mathbf{M} \ddot{\mathbf{q}}+\mathbf{N}=\mathbf{Q}
$$

where $\mathbf{M}=\mathbf{M}(\mathbf{q})$ is the $m \times m$ inertia matrix, $\mathbf{N}=\mathbf{N}(\mathbf{q}, \dot{\mathbf{q}})$ is the $m \times 1$ vector of Coriolis, centrifugal and gravitational forces, and $\mathbf{Q}$ is the $m \times 1$ vector of nonconservative generalized forces given by

$$
\mathbf{Q}=\left[\begin{array}{l}
\boldsymbol{\tau} \\
\mathbf{0}
\end{array}\right]
$$

The $m-n$ loop-closure constraint equations can be written in the form

$$
g_{j}(\mathbf{q})=0, \quad j=1, \cdots, m-n
$$

where $g_{j}$ 's are scalar functions. The equations of motion of the fully rigid parallel robot can then be expressed as

$$
\mathbf{M} \ddot{\mathbf{q}}+\mathbf{N}=\mathbf{Q}+\mathbf{A} \lambda
$$

where $\lambda=\left[\begin{array}{lll}\lambda_{1} & \cdots & \lambda_{m-n}\end{array}\right]^{\mathrm{T}}$ is the vector of Lagrange multipliers, and $\mathbf{A}$ is $m \times(m-n)$ matrix whose elements are given by

$$
A_{k j}=\frac{\partial g_{j}}{\partial q_{k}}, \quad j=1, \cdots, m-n, \quad k=1, \cdots, m
$$

Now consider the flexible-joint parallel robot. The gear ratios $R_{i}$ are assumed to be large enough so that the rotational kinetic energy of each rotor is approximately due only to its own spin [13]. By this assumption, the coupling terms between the joint and actuator accelerations disappear and the equations of motion of the flexible-joint parallel robot can be obtained as $[16,17]$

$$
\begin{aligned}
& \boldsymbol{\tau}=\mathbf{J} \ddot{\boldsymbol{\theta}}+\mathbf{C}\left(\dot{\boldsymbol{\theta}}-\dot{\mathbf{q}}^{\mathrm{a}}\right)+\mathbf{K}\left(\boldsymbol{\theta}-\mathbf{q}^{\mathrm{a}}\right) \\
& \mathbf{M} \ddot{\mathbf{q}}+\mathbf{N}=\mathbf{T}^{\mathrm{d}}+\mathbf{T}^{\mathrm{s}}+\mathbf{A} \boldsymbol{\lambda}
\end{aligned}
$$


where $\mathbf{J}=\operatorname{diag}\left(J_{1} R_{1}^{2}, \cdots, J_{n} R_{n}^{2}\right)$ is the $n \times n$ diagonal matrix of effective polar mass moments of inertia of the rotors at the output shafts of their connected gearboxes, and $\mathbf{C}=\operatorname{diag}\left(c_{1}, \cdots, c_{n}\right)$ and $\mathbf{K}=\operatorname{diag}\left(k_{1}, \cdots, k_{n}\right)$ are, respectively, the $n \times n$ damping and stiffness matrices of the actuated joints. The $m \times 1$ vectors of torques transmitted to the links through the dampers and springs are given in order as follows:

$$
\begin{aligned}
\mathbf{T}^{\mathrm{d}} & =\left[\begin{array}{c}
\mathbf{C}\left(\dot{\boldsymbol{\theta}}-\dot{\mathbf{q}}^{\mathrm{a}}\right) \\
\mathbf{0}
\end{array}\right] \\
\mathbf{T}^{\mathrm{s}} & =\left[\begin{array}{c}
\mathbf{K}\left(\boldsymbol{\theta}-\mathbf{q}^{\mathrm{a}}\right) \\
\mathbf{0}
\end{array}\right]
\end{aligned}
$$

\section{Computation of Input Torques and their Boundedness near Drive Singularities}

The inverse kinematic analysis of a flexible-joint robot is performed in the same manner as of its rigid-joint counterpart [16, 20]. Given a task, assume that the inverse kinematic solution for it does not contain any singularities, and the time histories of the link variables $q_{1}, \cdots, q_{m}$ and their first- and second-order time derivatives are obtained for the prescribed vector of task variables $\mathbf{x}(t)=\left[\begin{array}{lll}x_{1}(t) & \cdots & x_{n}(t)\end{array}\right]^{\mathrm{T}}$. Here $t$ denotes the time variable. The inverse kinematic singularities of closed-loop chains are in general encountered at the boundaries of the workspace [5]. For this reason, they are not a major concern and are left out of the scope of this study. Now partition the matrices $\mathbf{M}$ and $\mathbf{A}$ and the vector $\mathbf{N}$ as

$$
\begin{aligned}
& \mathbf{M}=\left[\begin{array}{c}
\mathbf{M}_{n \times m}^{\mathrm{a}} \\
\mathbf{M}_{(m-n) \times m}^{\mathrm{u}}
\end{array}\right] \\
& \mathbf{A}=\left[\begin{array}{c}
\mathbf{A}_{n \times(m-n)}^{\mathrm{a}} \\
\mathbf{A}_{(m-n) \times(m-n)}^{\mathrm{u}}
\end{array}\right] \\
& \mathbf{N}=\left[\begin{array}{c}
\mathbf{N}_{n \times 1}^{\mathrm{a}} \\
\mathbf{N}_{(m-n) \times 1}^{\mathrm{u}}
\end{array}\right]
\end{aligned}
$$


Equation (8) can then be expressed as the following two equations:

$\mathbf{C}\left(\dot{\boldsymbol{\theta}}-\dot{\mathbf{q}}^{\mathrm{a}}\right)+\mathbf{K}\left(\boldsymbol{\theta}-\mathbf{q}^{\mathrm{a}}\right)=\mathbf{M}^{\mathrm{a}} \ddot{\mathbf{q}}+\mathbf{N}^{\mathrm{a}}-\mathbf{A}^{\mathrm{a} \lambda}$

$\mathbf{A}^{\mathrm{u}} \lambda=\mathbf{M}^{\mathrm{u}} \ddot{\mathbf{q}}+\mathbf{N}^{\mathrm{u}}$

Having $\mathbf{q}, \dot{\mathbf{q}}$ and $\ddot{\mathbf{q}}$ obtained as explained above, unless the square submatrix $\mathbf{A}^{\mathrm{u}}$ is singular, the Lagrange multipliers can be computed by rearranging equation (15) as

$\lambda=\left(\mathbf{A}^{\mathrm{u}}\right)^{-1}\left(\mathbf{M}^{\mathrm{u}} \ddot{\mathbf{q}}+\mathbf{N}^{\mathrm{u}}\right)$

If all damping coefficients are nonzero, then matrix $\mathbf{C}$ is invertible and equation (14) is a system of $n$ first-order differential equations for the variable $\boldsymbol{\theta}$ of the form

$$
\dot{\boldsymbol{\theta}}=\dot{\mathbf{q}}^{\mathrm{a}}+\mathbf{C}^{-1}\left[\mathbf{M}^{\mathrm{a}} \ddot{\mathbf{q}}+\mathbf{N}^{\mathrm{a}}-\mathbf{A}^{\mathrm{a}} \boldsymbol{\lambda}-\mathbf{K}\left(\boldsymbol{\theta}-\mathbf{q}^{\mathrm{a}}\right)\right]
$$

Thus, $\boldsymbol{\theta}$ can be calculated by numerically integrating equation (17), and $\ddot{\boldsymbol{\theta}}$ can be obtained by numerical differentiation. Finally, the time history of the vector $\tau$ can be computed from equation (7).

If some of the damping coefficients are negligibly small and taken as zero in the dynamic model, then matrix $\mathbf{C}$ is singular and equation (14) is a system of differential-algebraic equations for the variable $\boldsymbol{\theta}$. However, these equations are uncoupled since the off-diagonal elements of the matrices $\mathbf{C}$ and $\mathbf{K}$ are all zero. Therefore, a nonzero damping coefficient $c_{\beta}$ leads to a first-order differential equation of the form

$$
\dot{\theta}_{\beta}=\dot{q}_{\beta}^{\mathrm{a}}+\frac{1}{c_{\beta}}\left[\sum_{k=1}^{m} M_{\beta k}^{\mathrm{a}} \ddot{q}_{k}+N_{\beta}^{\mathrm{a}}-\sum_{j=1}^{m-n} A_{\beta j}^{\mathrm{a}} \lambda_{j}-k_{\beta}\left(\theta_{\beta}-q_{\beta}^{\mathrm{a}}\right)\right]
$$

A zero damping coefficient $c_{\mu}$, on the other hand, leads to an algebraic equation which can be solved for $\theta_{\mu}$ as follows:

$$
\theta_{\mu}=q_{\mu}^{\mathrm{a}}+\frac{1}{k_{\mu}}\left(\sum_{k=1}^{m} M_{\mu k}^{\mathrm{a}} \ddot{q}_{k}+N_{\mu}^{\mathrm{a}}-\sum_{j=1}^{m-n} A_{\mu j}^{\mathrm{a}} \lambda_{j}\right)
$$

If all of the damping coefficients are assumed to be zero, then equation (14) is a system of algebraic equations whose solution is

$$
\boldsymbol{\theta}=\mathbf{q}^{\mathrm{a}}+\mathbf{K}^{-1}\left(\mathbf{M}^{\mathrm{a}} \ddot{\mathbf{q}}+\mathbf{N}^{\mathrm{a}}-\mathbf{A}^{\mathrm{a}} \boldsymbol{\lambda}\right)
$$

We present below also a generalized alternative method that can be used for any case of structural damping. 
Writing equation (14) at time $t_{v+1}$ and rearranging yields

$$
\begin{aligned}
\mathbf{C} \dot{\boldsymbol{\theta}}\left(t_{v+1}\right)+\mathbf{K} \boldsymbol{\theta}\left(t_{v+1}\right)= & \mathbf{C} \dot{\mathbf{q}}^{\mathrm{a}}\left(t_{v+1}\right)+\mathbf{K} \mathbf{q}^{\mathrm{a}}\left(t_{v+1}\right)+\mathbf{M}^{\mathrm{a}}\left(\mathbf{q}\left(t_{v+1}\right)\right) \ddot{\mathbf{q}}\left(t_{v+1}\right) \\
& +\mathbf{N}^{\mathrm{a}}\left(\mathbf{q}\left(t_{v+1}\right), \dot{\mathbf{q}}\left(t_{v+1}\right)\right)-\mathbf{A}^{\mathrm{a}}\left(\mathbf{q}\left(t_{v+1}\right)\right) \lambda\left(t_{v+1}\right)
\end{aligned}
$$

By using the backward-difference method, we have

$$
\dot{\boldsymbol{\theta}}\left(t_{v+1}\right)=\frac{1}{h}\left[\boldsymbol{\theta}\left(t_{v+1}\right)-\boldsymbol{\theta}\left(t_{v}\right)\right]
$$

or solving for $\boldsymbol{\theta}\left(t_{v+1}\right)$,

$$
\boldsymbol{\theta}\left(t_{v+1}\right)=\boldsymbol{\theta}\left(t_{v}\right)+h \dot{\boldsymbol{\theta}}\left(t_{v+1}\right)
$$

Here, $h$ is the time-step size. Substituting equation (23) into equation (21) and solving for $\dot{\boldsymbol{\theta}}\left(t_{v+1}\right)$ gives

$$
\begin{aligned}
\dot{\boldsymbol{\theta}}\left(t_{v+1}\right)= & (\mathbf{C}+h \mathbf{K})^{-1}\left\{\mathbf{C} \dot{\mathbf{q}}^{\mathrm{a}}\left(t_{v+1}\right)+\mathbf{M}^{\mathrm{a}}\left(\mathbf{q}\left(t_{v+1}\right)\right) \ddot{\mathbf{q}}\left(t_{v+1}\right)\right. \\
& +\mathbf{N}^{\mathrm{a}}\left(\mathbf{q}\left(t_{v+1}\right), \dot{\mathbf{q}}\left(t_{v+1}\right)\right)-\mathbf{A}^{\mathrm{a}}\left(\mathbf{q}\left(t_{v+1}\right)\right) \lambda\left(t_{v+1}\right) \\
& \left.-\mathbf{K}\left[\boldsymbol{\theta}\left(t_{v}\right)-\mathbf{q}^{\mathrm{a}}\left(t_{v+1}\right)\right]\right\}
\end{aligned}
$$

Drive singularities are the configurations where the matrix $\mathbf{A}^{\mathrm{u}}$ is not of full rank. Near these configurations, the Lagrange multipliers, and hence the required input torques for realizing the prescribed task, are in general unbounded. In order to have a bounded inverse dynamics solution, the Lagrange multipliers should all have finite limits as the singularity is approached. Let it be assumed that $\mathbf{A}^{\mathrm{u}}$ is rank-deficient by one at the singularity time $t_{\mathrm{s}}$, which is generally the case $[6,7]$.

Equation (16) can be rewritten in terms of the adjoint and determinant of $\mathbf{A}^{\mathrm{u}}$ as

$$
\lambda=\frac{1}{\operatorname{det}\left(\mathbf{A}^{\mathrm{u}}\right)} \operatorname{adj}\left(\mathbf{A}^{\mathrm{u}}\right)\left(\mathbf{M}^{\mathrm{u}} \ddot{\mathbf{q}}+\mathbf{N}^{\mathrm{u}}\right)
$$

When the robot is away from a drive singularity, the matrix $\mathbf{A}^{\mathrm{u}}$ and its adjoint matrix have full rank, and its determinant is nonzero. Therefore, the Lagrange multipliers can be regularly determined from equation (25). But when the determinant of $\mathbf{A}^{\mathrm{u}}$ vanishes at time $t=t_{\mathrm{s}}$, equation (25) results in divisions by zero. Thus, at least one of the limits $\lim _{t \rightarrow t_{\mathrm{s}}} \lambda_{j}(t), j=1, \cdots, m-n$, is not finite unless the following condition is satisfied at the singular position:

$$
\operatorname{adj}\left(\mathbf{A}^{\mathrm{u}}\right)\left(\mathbf{M}^{\mathrm{u}} \ddot{\mathbf{q}}+\mathbf{N}^{\mathrm{u}}\right)=\mathbf{0}
$$


Satisfaction of equation (26) ensures that the set of equations given by equation (15) is consistent at the singularity time. If equation (26) holds and additionally the first-order time derivative of the determinant of $\mathbf{A}^{\mathrm{u}}$ does not vanish at time $t_{\mathrm{s}}$, then all limits $\lim _{t \rightarrow t_{\mathrm{s}}} \lambda_{j}(t)$ are finite and can be evaluated using l'Hôpital's Rule.

This implies that the required motor torques will remain bounded in the neighborhood of the singularity. However, although having finite limits as $t \rightarrow t_{\mathrm{s}}$, the Lagrange multipliers are not defined at $t=t_{\mathrm{s}}$. These discontinuities can be removed by redefining $\lambda$ at $t=t_{\mathrm{s}}$ such that $\lambda\left(t_{\mathrm{s}}\right)=\lim _{t \rightarrow t_{\mathrm{s}}} \lambda(t)$. The differentiability of the functions required in the application of l'Hôpital's Rule can be ensured by using a smooth trajectory generated with a polynomial function of time. Before concluding this section, it is worth mentioning that although equation (26) gives $m-n$ equations to be satisfied at the singular configuration, $m-n-1$ of them are redundant. This is because the rank of the adjoint matrix of a square matrix $\mathbf{P}$ is 1 if $\mathbf{P}$ is rank-deficient by one [21]. Consequently, when $\mathbf{A}^{\mathrm{u}}$ loses one rank, all the individual equations in matrix equation (26) equivalently describe the same unique consistency condition. In the next section, we propose a novel method for planning a trajectory satisfying this condition. Our method also features two distinct tests: one for the nonzeroness of the first-order time derivative of the determinant of $\mathbf{A}^{\mathrm{u}}$ at the singularity time, and one for preventing undesired backand-forth motion of the endpoint. For the latter test, four additional conditions are derived, and in this manner, the possibility of unintended multiple occurrences of the same singularity is avoided.

\section{A Novel Trajectory Planning Method}

The previous section deals with the problem of removability of drive singularities of parallel robots in the presence of joint flexibility. It may be useful to note that drive singularities and the conditions under which they become removable would be identical for a flexible-joint parallel robot and its fully rigid counterpart since equation (15) does not contain any element of $\boldsymbol{\theta}$, or of its time derivatives. However, trajectory planning of flexible-joint parallel robots is quite different from and more complex than that of fully rigid parallel robots. This is because due to the elastic media at the transmission, the motors cannot instantaneously change the accelerations of the end-effector $[15,16]$. Motivated by this fact, in this section, we propose a new method of planning singularity-removed trajectories for flexible-joint parallel robots.

In the absence of obstacles, the optimal path between two points is the straight line joining these points. For this reason, we will focus here on tasks where the endpoint $E$ of the robot has to perform a rest-to-rest motion along a straight line 
from an initial position $\left(x_{E^{\prime}}, y_{E^{\prime}}, z_{E^{\prime}}\right)$ to a final position $\left(x_{E^{\prime \prime}}, y_{E^{\prime \prime}}, z_{E^{\prime \prime}}\right)$ in a prescribed time $t_{\mathrm{f}}$. Such tasks constitute the majority of pick-and-place applications. The endpoint trajectory can be expressed as

$$
\begin{aligned}
& x_{E}(t)=x_{E^{\prime}}+\left(x_{E^{\prime \prime}}-x_{E^{\prime}}\right) f(t) \\
& y_{E}(t)=y_{E^{\prime}}+\left(y_{E^{\prime \prime}}-y_{E^{\prime}}\right) f(t) \\
& z_{E}(t)=z_{E^{\prime}}+\left(z_{E^{\prime \prime}}-z_{E^{\prime}}\right) f(t)
\end{aligned}
$$

where $f(t)$ is a timing function such that

$$
\begin{aligned}
& f(0)=0 \\
& f\left(t_{\mathrm{f}}\right)=1
\end{aligned}
$$

when the initial time is taken to be $t=0$. For a flexible-joint robot, the input torques instantaneously affect the snaps of the end-effector rather than its accelerations $[15,16]$. Hence, for the manipulator to be at rest at the start and end of the motion, the following eight conditions are required:

$$
\begin{aligned}
& \dot{f}(0)=\ddot{f}(0)=\dddot{f}(0)=f^{(4)}(0)=0 \\
& \dot{f}\left(t_{\mathrm{f}}\right)=\ddot{f}\left(t_{\mathrm{f}}\right)=\dddot{f}\left(t_{\mathrm{f}}\right)=f^{(4)}\left(t_{\mathrm{f}}\right)=0
\end{aligned}
$$

Let the drive singular configuration to be passed through during the execution of the trajectory correspond to a value of $f$ equal to $f_{\mathrm{s}}$. So we have

$$
f\left(t_{\mathrm{s}}\right)=f_{\mathrm{s}}
$$

where $t_{\mathrm{s}}$ is the singularity time, which will be set to an appropriate value in the interval $\left(0, t_{\mathrm{f}}\right)$. Notice that $0<f_{\mathrm{s}}<1$, and recall our assumption that $\operatorname{rank}\left(\mathbf{A}^{\mathrm{u}}\left(t_{\mathrm{s}}\right)\right)=m-n-1$. Since the consistency condition that should be satisfied at the singular configuration of interest contains first-order acceleration and second-order velocity terms, it can be written as

$$
\kappa_{1}\left[\dot{f}\left(t_{\mathrm{s}}\right)\right]^{2}+\kappa_{2} \ddot{f}\left(t_{\mathrm{s}}\right)+\kappa_{3}=0
$$

where $\kappa_{1}, \kappa_{2}$ and $\kappa_{3}$ are constants which can be determined using the values of the robot parameters together with the values of the link position variables at that singular configuration.

Since there are totally 12 equations to be satisfied, the timing function is selected to be an eleventh-degree polynomial function of the form 


$$
f(t)=\sum_{p=0}^{11} a_{p} t^{p}
$$

It follows from equations (30) and (32) that the first five coefficients $a_{0}, a_{1}, a_{2}$, $a_{3}$ and $a_{4}$ are all equal to zero. Thus, the polynomial $f$ can be factored as

$$
f(t)=t^{5}\left(a_{5}+a_{6} t+a_{7} t^{2}+a_{8} t^{3}+a_{9} t^{4}+a_{10} t^{5}+a_{11} t^{6}\right)
$$

For a chosen value of $t_{\mathrm{s}}$, equations (31), (33), and (34) constitute a system of 6 equations that are linear in the remaining 7 unknowns $a_{5}, a_{6}, a_{7}, a_{8}, a_{9}, a_{10}$ and $a_{11}$. By treating $a_{11}$ as a parameter, the solution of this system can be expressed as

$$
\begin{aligned}
& a_{5}=\frac{1}{\left(t_{\mathrm{f}}-t_{\mathrm{s}}\right)^{5}}\left[\frac{t_{\mathrm{f}}^{5} f_{\mathrm{s}}}{t_{\mathrm{s}}^{5}}-\frac{\left(126 t_{\mathrm{s}}^{4}-560 t_{\mathrm{s}}^{3} t_{\mathrm{f}}+945 t_{\mathrm{s}}{ }^{2} t_{\mathrm{f}}{ }^{2}-720 t_{\mathrm{s}} t_{\mathrm{f}}^{3}+210 t_{\mathrm{f}}{ }^{4}\right) t_{\mathrm{s}}}{t_{\mathrm{f}}^{5}}\right] \\
& +t_{\mathrm{s}} t_{\mathrm{f}}^{5} a_{11} \\
& a_{6}=\frac{5}{\left(t_{\mathrm{f}}-t_{\mathrm{s}}\right)^{5}}\left[\frac{84 t_{\mathrm{s}}^{5}-350 t_{\mathrm{s}}^{4} t_{\mathrm{f}}+525 t_{\mathrm{s}}^{3} t_{\mathrm{f}}^{2}-300 t_{\mathrm{s}}^{2} t_{\mathrm{f}}{ }^{3}+42 t_{\mathrm{f}}^{5}}{t_{\mathrm{f}}^{6}}-\frac{t_{\mathrm{f}}^{4} f_{\mathrm{s}}}{t_{\mathrm{s}}^{5}}\right. \\
& \left.+\frac{\left(5 t_{\mathrm{s}}^{6}-24 t_{\mathrm{s}}^{5} t_{\mathrm{f}}+45 t_{\mathrm{s}}^{4} t_{\mathrm{f}}^{2}-40 t_{\mathrm{s}}^{3} t_{\mathrm{f}}^{3}+15 t_{\mathrm{s}}^{2} t_{\mathrm{f}}^{4}-t_{\mathrm{f}}^{6}\right) t_{\mathrm{f}}^{4} a_{11}}{5}\right] \\
& a_{7}=\frac{10}{\left(t_{\mathrm{f}}-t_{\mathrm{s}}\right)^{5}}\left[\frac{t_{\mathrm{f}}^{3} f_{\mathrm{s}}}{t_{\mathrm{s}}^{5}}-\frac{54 t_{\mathrm{s}}^{5}-200 t_{\mathrm{s}}^{4} t_{\mathrm{f}}+225 t_{\mathrm{s}}^{3} t_{\mathrm{f}}{ }^{2}-150 t_{\mathrm{s}} t_{\mathrm{f}}^{4}+72 t_{\mathrm{f}}{ }^{5}}{t_{\mathrm{f}}^{7}}\right. \\
& \left.-\frac{\left(2 t_{\mathrm{s}}^{6}-9 t_{\mathrm{s}}^{5} t_{\mathrm{f}}+15 t_{\mathrm{s}}{ }^{4} t_{\mathrm{f}}{ }^{2}-10 t_{\mathrm{s}}^{3} t_{\mathrm{f}}^{3}+3 t_{\mathrm{s}} t_{\mathrm{f}}^{5}-t_{\mathrm{f}}{ }^{6}\right) t_{\mathrm{f}}{ }^{3} a_{11}}{2}\right] \\
& a_{8}=\frac{5}{\left(t_{\mathrm{f}}-t_{\mathrm{s}}\right)^{5}}\left[\frac{63 t_{\mathrm{s}}^{5}-175 t_{\mathrm{s}}^{4} t_{\mathrm{f}}+450 t_{\mathrm{s}}^{2} t_{\mathrm{f}}{ }^{3}-525 t_{\mathrm{s}} t_{\mathrm{f}}{ }^{4}+189 t_{\mathrm{f}}{ }^{5}}{t_{\mathrm{f}}^{8}}-\frac{2 t_{\mathrm{f}}{ }^{2} f_{\mathrm{s}}}{t_{\mathrm{s}}^{5}}\right. \\
& \left.+2\left(t_{\mathrm{s}}^{6}-4 t_{\mathrm{s}}^{5} t_{\mathrm{f}}+5 t_{\mathrm{s}}^{4} t_{\mathrm{f}}{ }^{2}-5 t_{\mathrm{s}}{ }^{2} t_{\mathrm{f}}{ }^{4}+4 t_{\mathrm{s}} t_{\mathrm{f}}^{5}-t_{\mathrm{f}}{ }^{6}\right) t_{\mathrm{f}}{ }^{2} a_{11}\right] \\
& a_{9}=\frac{5}{\left(t_{\mathrm{f}}-t_{\mathrm{s}}\right)^{5}}\left[\frac{t_{\mathrm{f}} f_{\mathrm{s}}}{t_{\mathrm{s}}^{5}}-\frac{14 t_{\mathrm{s}}{ }^{5}-175 t_{\mathrm{s}}{ }^{3} t_{\mathrm{f}}{ }^{2}+400 t_{\mathrm{s}}{ }^{2} t_{\mathrm{f}}{ }^{3}-350 t_{\mathrm{s}} t_{\mathrm{f}}{ }^{4}+112 t_{\mathrm{f}}{ }^{5}}{t_{\mathrm{f}}{ }^{9}}\right. \\
& \left.-\left(t_{\mathrm{s}}^{6}-3 t_{\mathrm{s}}^{5} t_{\mathrm{f}}+10 t_{\mathrm{s}}^{3} t_{\mathrm{f}}^{3}-15 t_{\mathrm{s}}{ }^{2} t_{\mathrm{f}}^{4}+9 t_{\mathrm{s}} t_{\mathrm{f}}^{5}-2 t_{\mathrm{f}}^{6}\right) t_{\mathrm{f}} a_{11}\right]
\end{aligned}
$$




$$
\begin{aligned}
a_{10} & =\frac{1}{\left(t_{\mathrm{f}}-t_{\mathrm{s}}\right)^{5}}\left[\frac{70 t_{\mathrm{s}}^{4}-315 t_{\mathrm{s}}^{3} t_{\mathrm{f}}+540 t_{\mathrm{s}}{ }^{2} t_{\mathrm{f}}{ }^{2}-420 t_{\mathrm{s}} t_{\mathrm{f}}{ }^{3}+126 t_{\mathrm{f}}{ }^{4}}{t_{\mathrm{f}}^{9}}-\frac{f_{\mathrm{s}}}{t_{\mathrm{s}}^{5}}\right. \\
& \left.+\left(t_{\mathrm{s}}{ }^{6}-15 t_{\mathrm{s}}{ }^{4} t_{\mathrm{f}}{ }^{2}+40 t_{\mathrm{s}}{ }^{3} t_{\mathrm{f}}{ }^{3}-45 t_{\mathrm{s}}{ }^{2} t_{\mathrm{f}}{ }^{4}+24 t_{\mathrm{s}} t_{\mathrm{f}}{ }^{5}-5 t_{\mathrm{f}}{ }^{6}\right) a_{11}\right]
\end{aligned}
$$

By substituting these coefficients into the expressions for the first two time derivatives of $f$ and rearranging, equation (35) can be simplified to

$\gamma_{2} a_{11}^{2}+\gamma_{1} a_{11}+\gamma_{0}=0$

where

$$
\begin{aligned}
& \gamma_{2}=\left(t_{\mathrm{f}}-t_{\mathrm{s}}\right)^{12} t_{\mathrm{s}}^{12} t_{\mathrm{f}}^{18} \kappa_{1} \\
& \gamma_{1}=-10\left(t_{\mathrm{f}}-t_{\mathrm{s}}\right)^{6} t_{\mathrm{s}}{ }^{6} t_{\mathrm{f}}{ }^{9}\left[\left(14 t_{\mathrm{s}}{ }^{4}-70 t_{\mathrm{s}}{ }^{3} t_{\mathrm{f}}+135 t_{\mathrm{s}}{ }^{2} t_{\mathrm{f}}{ }^{2}-120 t_{\mathrm{s}} t_{\mathrm{f}}{ }^{3}+42 t_{\mathrm{f}}{ }^{4}\right) \kappa_{1} t_{\mathrm{s}}{ }^{6}\right. \\
& \left.+\left(\kappa_{1} f_{\mathrm{s}}+\kappa_{2}\right)\left(t_{\mathrm{f}}-2 t_{\mathrm{s}}\right) t_{\mathrm{f}}{ }^{9}\right] \\
& \gamma_{0}=\left(25 \kappa_{1} f_{\mathrm{s}}^{2}+20 \kappa_{2} f_{\mathrm{s}}+\kappa_{3} t_{\mathrm{s}}^{2}\right) t_{\mathrm{f}}^{20}-\left(100 \kappa_{1} f_{\mathrm{s}}^{2}+90 \kappa_{2} f_{\mathrm{s}}+2 \kappa_{3} t_{\mathrm{s}}^{2}\right) t_{\mathrm{s}} t_{\mathrm{f}}{ }^{19} \\
& +\left(100 \kappa_{1} f_{\mathrm{s}}^{2}+90 \kappa_{2} f_{\mathrm{s}}+\kappa_{3} t_{\mathrm{s}}^{2}\right) t_{\mathrm{s}}{ }^{2} t_{\mathrm{f}}{ }^{18}+\left(2100 \kappa_{1} f_{\mathrm{s}}+2100 \kappa_{2}\right) t_{\mathrm{s}}{ }^{6} t_{\mathrm{f}}{ }^{14} \\
& -\left(10200 \kappa_{1} f_{\mathrm{s}}+9540 \kappa_{2}\right) t_{\mathrm{s}} t_{\mathrm{f}}^{13}+\left(18750 \kappa_{1} f_{\mathrm{s}}+17100 \kappa_{2}\right) t_{\mathrm{s}}^{8} t_{\mathrm{f}}{ }^{12} \\
& -\left(17000 \kappa_{1} f_{\mathrm{s}}+15350 \kappa_{2}\right) t_{\mathrm{s}}^{9} t_{\mathrm{f}}^{11}+\left(7700 \kappa_{1} f_{\mathrm{s}}+6930 \kappa_{2}\right) t_{\mathrm{s}}^{10} t_{\mathrm{f}}{ }^{10} \\
& -\left(1400 \kappa_{1} f_{\mathrm{s}}+1260 \kappa_{2}\right) t_{\mathrm{s}}{ }^{11} t_{\mathrm{f}}{ }^{9}+\left(44100 t_{\mathrm{f}}{ }^{8}-252000 t_{\mathrm{s}} t_{\mathrm{f}}{ }^{7}\right. \\
& +643500 t_{\mathrm{s}}{ }^{2} t_{\mathrm{f}}^{6}-957000 t_{\mathrm{s}}{ }^{3} t_{\mathrm{f}}^{5}+905025 t_{\mathrm{s}}{ }^{4} t_{\mathrm{f}}{ }^{4}-556500 t_{\mathrm{s}}{ }^{5} t_{\mathrm{f}}{ }^{3} \\
& \left.+217000 t_{\mathrm{s}}{ }^{6} t_{\mathrm{f}}{ }^{2}-49000 t_{\mathrm{s}}{ }^{7} t_{\mathrm{f}}+4900 t_{\mathrm{s}}{ }^{8}\right) \kappa_{1} t_{\mathrm{s}}{ }^{12}
\end{aligned}
$$

The solution of the quadratic equation (44) is

$a_{11}=\frac{-\gamma_{1} \pm \sqrt{\Delta_{1}}}{2 \gamma_{2}}$

where

$$
\Delta_{1}=\gamma_{1}^{2}-4 \gamma_{0} \gamma_{2}
$$

It is obvious that in order for the roots given by equation (48) to be real, the discriminant $\Delta_{1}$ must be nonnegative. Now, we propose the following method to obtain the coefficients of a rest-to-rest polynomial of the form of equation (37) that ensures the consistency of the robot's dynamic equations at a user-selected singularity crossing time:

Step 1) Select a $t_{\mathrm{s}} \in\left(0, t_{\mathrm{f}}\right)$ such that $\Delta_{1} \geq 0$.

Step 2) Compute the two $a_{11}$ 's from equation (48) 
Step 3) If $\Delta_{1}>0$, select and proceed with any one of the two distinct $a_{11}$ 's.

Step 4) Compute $a_{5}, a_{6}, a_{7}, a_{8}, a_{9}$ and $a_{10}$ from equations (38)-(43).

If in addition to the consistency, the first-order time derivative of $\operatorname{det}\left(\mathbf{A}^{\mathrm{u}}\right)$ is nonzero at $t_{\mathrm{s}}$, then the inverse dynamics solution is bounded as $t \rightarrow t_{\mathrm{s}}$. Therefore, after the trajectory polynomial is obtained using the above method, this second condition should be checked to guarantee the boundedness of the required input torques near $t_{\mathrm{s}}$.

Another important consideration in trajectory planning is the prevention of any undesired back-and-forth motion of the endpoint [11]. This is important not only for the efficiency of the robot [12], but also for avoiding possible additional occurrences of the same singularity at times other than the selected $t_{\mathrm{s}}$. Such unintended occurrences make it, in general, impossible to realize the trajectory since at these times the dynamic equations will be, in general, inconsistent. It is a fact that if $\dot{f}(t)$ is negative during a time interval, then the endpoint performs a backward motion in that interval. It follows from this fact that avoidance of any undesired backward motion requires the nonnegativity of $\dot{f}(t)$ over the time interval $\left(0, t_{\mathrm{f}}\right)$. Time differentiation of equation (37) gives

$$
\dot{f}(t)=t^{4}\left(5 a_{5}+6 a_{6} t+7 a_{7} t^{2}+8 a_{8} t^{3}+9 a_{9} t^{4}+10 a_{10} t^{5}+11 a_{11} t^{6}\right)
$$

As seen in equation (50), $t^{4}$ is a factor of the tenth-degree polynomial $\dot{f}(t)$. This could also be deduced directly from equation (32). Similarly, it follows from equation (33) that $\left(t-t_{\mathrm{f}}\right)^{4}$ is another factor of $\dot{f}(t)$. Thus, we have

$$
\dot{f}(t)=t^{4}\left(t-t_{\mathrm{f}}\right)^{4} b(t)
$$

where $b(t)$ is a second-degree polynomial of the form

$$
b(t)=b_{0}+b_{1} t+b_{2} t^{2}
$$

A term-by-term comparison of equations (50) and (51) yields

$$
\begin{aligned}
& b_{0}=9 a_{9}+40 t_{\mathrm{f}} a_{10}+110 t_{\mathrm{f}}^{2} a_{11} \\
& b_{1}=10 a_{10}+44 t_{\mathrm{f}} a_{11} \\
& b_{2}=11 a_{11}
\end{aligned}
$$


The factors $t^{4}$ and $\left(t-t_{\mathrm{f}}\right)^{4}$ are positive in the interval $\left(0, t_{\mathrm{f}}\right)$, which implies that $\operatorname{sgn}(\dot{f}(t))=\operatorname{sgn}(b(t))$ for all $t$ in that interval. The discriminant of the quadratic polynomial $b(t)$ is

$\Delta_{2}=b_{1}^{2}-4 b_{0} b_{2}$

It can then be concluded that $b(t)$, and hence also $\dot{f}(t)$, is nonnegative $\forall t \in\left(0, t_{\mathrm{f}}\right)$ if and only if one of the following four statements holds:

(i) $b_{2}>0$ and $\Delta_{2} \leq 0$;

(ii) $b_{2}>0, \Delta_{2}>0$ and $\frac{-b_{1}+\sqrt{\Delta_{2}}}{2 b_{2}} \leq 0$;

(iii) $b_{2}>0, \Delta_{2}>0$ and $\frac{-b_{1}-\sqrt{\Delta_{2}}}{2 b_{2}} \geq t_{\mathrm{f}}$;

(iv) $b_{2}<0, \Delta_{2}>0, \frac{-b_{1}+\sqrt{\Delta_{2}}}{2 b_{2}} \leq 0$ and $\frac{-b_{1}-\sqrt{\Delta_{2}}}{2 b_{2}} \geq t_{\mathrm{f}}$.

Consequently, once the polynomial coefficients $a_{p}$ are computed using the proposed method, another check, which is for preventing any undesired back-andforth motion of the endpoint, is to ensure that one of the above statements (i)-(iv) is true. If one or both of the two aforementioned checks is not satisfied, then a new trajectory can be planned by restarting from Step 3 with the other distinct $a_{11}$ value (if exists) that has not been used yet, or from Step 1 with a new choice of $t_{\mathrm{s}}$.

\section{Case Study}

Let us consider a planar 5R parallel robot, as shown in Figure 1, where $\mathrm{R}$ denotes revolute joint. Each moving link $w(w=1,2,3,4)$ is modeled as a uniform homogeneous slender rod of length $r_{w}$ and mass $m_{w}$. The origin of the fixed rectangular Cartesian coordinate system with axes $x$ and $y$ is located at joint $A$, and joint $C$ is at $\left(r_{0}, 0\right)$. The gravitational acceleration vector is normal to the $x y$-plane. The robot has two rigid degrees of freedom. Thus, there are two motors, the first of which is located at joint $A$ and the second at joint $C$. However, due to the flexibility of the actuated joints, the total number of degrees 
of freedom is equal to 4 . The vector of angular positions of the output shafts of the motor gearboxes is $\boldsymbol{\theta}=\left[\begin{array}{ll}\theta_{1} & \theta_{2}\end{array}\right]^{\mathrm{T}}$ and the vector of link variables is

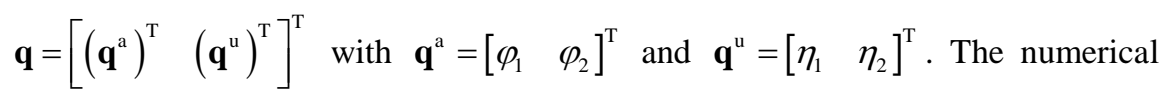
values of the model parameters are as follows: $m_{1}=m_{2}=m_{3}=m_{4}=12 \mathrm{~kg}$, $r_{0}=r_{1}=r_{2}=r_{3}=r_{4}=5 \mathrm{~m}, \quad J_{1}=J_{2}=5 \times 10^{-5} \mathrm{~kg} \cdot \mathrm{m}^{2}, \quad R_{1}=R_{2}=100$, $c_{1}=c_{2}=3.6 \mathrm{~N} \cdot \mathrm{m} \cdot \mathrm{s} / \mathrm{rad}, k_{1}=k_{2}=3600 \mathrm{~N} \cdot \mathrm{m} / \mathrm{rad}$.

The desired task requires the endpoint $E$ to move along a straight line that is parallel to the $y$-axis, starting from rest at time $t=0$ at position $E^{\prime}(2.5 \mathrm{~m}, 6.33 \mathrm{~m})$ (with $\varphi_{1}=115.6^{\circ}, \varphi_{2}=64.4^{\circ}, \eta_{1}=21.3^{\circ}$ and $\eta_{2}=158.7^{\circ}$ ) and ending at rest at time $t_{\mathrm{f}}=1 \mathrm{~s}$ at position $E^{\prime \prime}(2.5 \mathrm{~m}, 2.33 \mathrm{~m})$. The prescribed path of the endpoint intersects the drive singularity locus at point $(x, y)=(2.5,4.33) \mathrm{m}$ with $\varphi_{1}=120^{\circ}, \varphi_{2}=60^{\circ}, \eta_{1}=0^{\circ}$ and $\eta_{2}=180^{\circ}$. This intersection point is the midpoint of the path, that is, $f_{\mathrm{s}}=0.5$. It follows from equation (26) that the unique consistency condition which should be satisfied at this drive singular configuration is

$$
\begin{aligned}
& r_{4}\left[\frac{1}{2} m_{3} r_{1} r_{3} \ddot{\varphi}_{1} \cos \left(\varphi_{1}-\eta_{1}\right)+\frac{1}{3} m_{3} r_{3}^{2} \ddot{\eta}_{1}-\frac{1}{2} m_{3} r_{1} r_{3} \dot{\varphi}_{1}^{2} \sin \left(\varphi_{1}-\eta_{1}\right)\right]- \\
& r_{3}\left[\frac{1}{2} m_{4} r_{2} r_{4} \ddot{\varphi}_{2} \cos \left(\varphi_{2}-\eta_{2}\right)+\frac{1}{3} m_{4} r_{4}^{2} \ddot{\eta}_{2}-\frac{1}{2} m_{4} r_{2} r_{4} \dot{\varphi}_{2}^{2} \sin \left(\varphi_{2}-\eta_{2}\right)\right]=0
\end{aligned}
$$

If this equation holds and, in addition, the first-order time derivative of $\operatorname{det}\left(\mathbf{A}^{\mathrm{u}}\right)=r_{3} r_{4} \sin \left(\eta_{1}-\eta_{2}\right)$ is nonzero at $t_{\mathrm{s}}$, then the Lagrange multipliers $\lambda_{1}$ and $\lambda_{2}$ will both have finite limits as $t$ goes to $t_{\mathrm{s}}$. After some manipulations and substitution of the numerical values into equation (57), the condition to be satisfied by $f$ for consistency at the aforementioned encountered singular configuration is obtained in the form of equation (35) as

$$
\frac{320 \sqrt{3}}{3}\left[\dot{f}\left(t_{\mathrm{s}}\right)\right]^{2}-800 \ddot{f}\left(t_{\mathrm{s}}\right)=0
$$

Figure 2 shows the real roots of the quadratic equation (44) for different choices of $t_{\mathrm{s}}$ for the given task of the robot. 


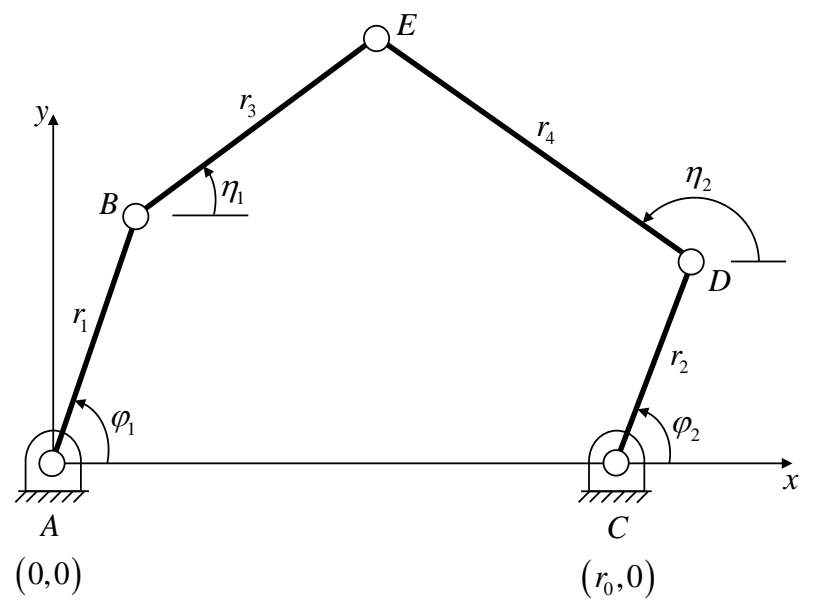

Figure 1

A planar $5 \mathrm{R}$ parallel robot

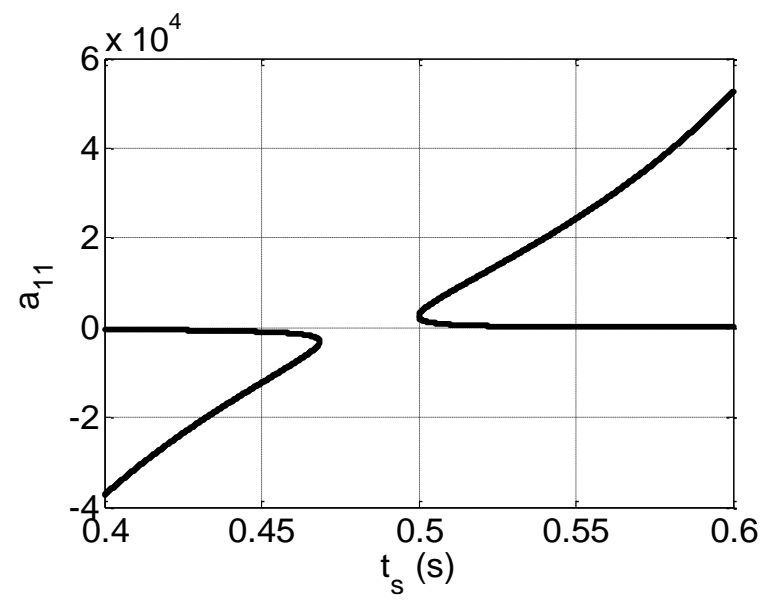

Figure 2

Values of $a_{11}$ calculated with different selections of $t_{\mathrm{s}}$ for the prescribed task of the robot

\subsection{Case 1: A Consistent Trajectory Yielding a Determinant That Has Flatness 2 at the Singularity Time}

In this first case, a consistent trajectory is planned by selecting $t_{\mathrm{s}}$ equal to $0.5 \mathrm{~s}$, which is half of the total task duration. This selection yields $\Delta_{1}=0$. Therefore, there is only one real value for $a_{11}$, which is $a_{11}=2520$. The remaining nonzero coefficients are then determined as: $a_{5}=1386, a_{6}=-9240, a_{7}=25740$, 
$a_{8}=-38115, \quad a_{9}=31570, \quad a_{10}=-13860$. These give $b_{2}=27720>0$ and $\Delta_{2}=0$. Thus, statement (i) holds true and there will be no back-and-forth motion of the endpoint. However, the first and second derivatives of the resulting $f$ function are both zero at the time $t=t_{\mathrm{s}}=0.5 \mathrm{~s}$ (see Figure 3). For this reason, the determinant of $\mathbf{A}^{\mathrm{u}}$ vanishes at this time together with its first two time derivatives. To the best knowledge of the authors, this is the first time that such a high-order drive singularity is exemplified for a flexible-joint parallel robot. The motor torques are not bounded near $t=t_{\mathrm{s}}$, as can be seen in Figure 4, although the consistency condition is satisfied.

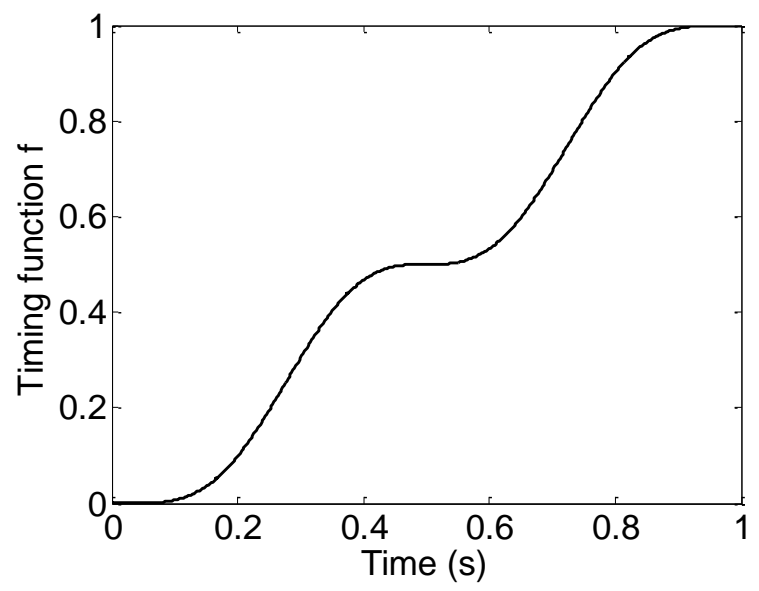

Figure 3

Timing function $f$ in Case 1

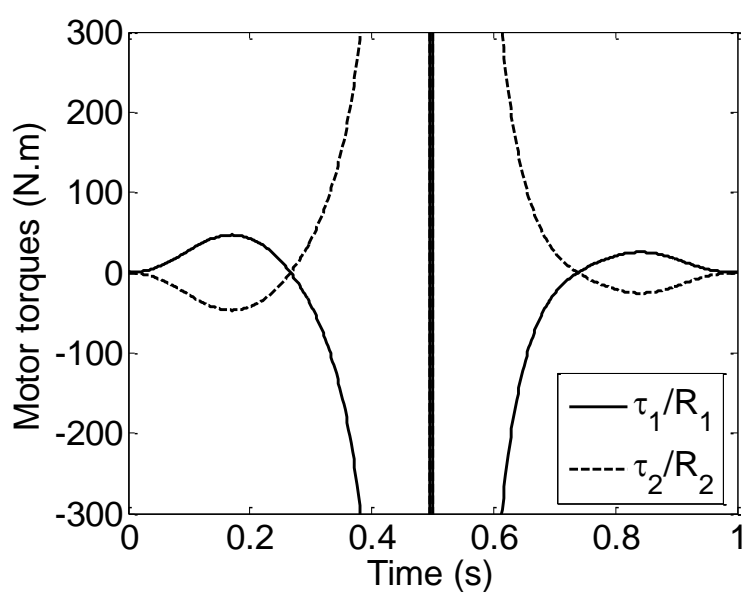

Figure 4

Motor torques required in Case 1 


\subsection{Case 2: A Consistent Trajectory That Results in Multiple Occurrences of the Same Singularity}

To overcome the problem encountered in Case $1, t_{\mathrm{s}}$ is slightly changed to $0.5005 \mathrm{~s}$, and a new consistent trajectory is planned. With the new value of $t_{\mathrm{s}}$, we have $\Delta_{1}=2.4229 \times 10^{-4}>0$. Hence, there are two distinct real solutions for $a_{11}$, namely $a_{11}=3315.47555494455$ and $a_{11}=1901.94295206846$. Choosing the first root $a_{11}=3315.47555494455$ leads to the following coefficients: $a_{5}=1784.13551062975, \quad a_{6}=-12026.1531080933, a_{7}=33698.7328810201$, $a_{8}=-50051.1106557429, a_{9}=41515.4331025939, a_{10}=-18235.5132853524$.

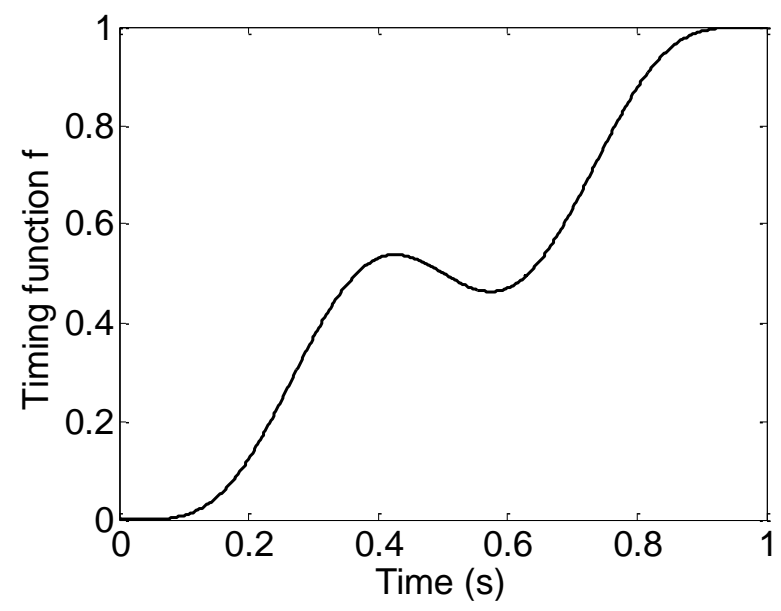

Figure 5

Timing function $f$ in Case 2

By examining the resulting polynomial function (Figure 5), it can be seen that the singularity corresponding to $f=0.5$ happens to occur at two additional times ( $t=0.3668 \mathrm{~s}$ and $t=0.6328 \mathrm{~s}$ ) other than the intended time of passing through its corresponding configuration $(t=0.5005 \mathrm{~s})$. This is because we have $b_{2}=36470.2311>0$ and $\Delta_{2}=2.9011 \times 10^{7}>0$, but $\frac{-b_{1}+\sqrt{\Delta_{2}}}{2 b_{2}}=0.5739>0$ and $\frac{-b_{1}-\sqrt{\Delta_{2}}}{2 b_{2}}=0.4262<t_{\mathrm{f}}=1$, which means that none of the statements (i)-(iv) holds true. Such a possibility of multiple occurrences of the same singularity was not considered before in the literature. The consistency condition is satisfied, while $\operatorname{det}\left(\mathbf{A}^{\mathrm{u}}\right)$ has a nonzero first-order time derivative at the intended 
singularity time $t=0.5005 \mathrm{~s}$. Thus, the inverse dynamics solution is bounded near this time. However, it is unbounded near the unintended singularity times $t=0.3668 \mathrm{~s}$ and $t=0.6328 \mathrm{~s}$ since the consistency condition is not fulfilled at these instants. The time histories of the required motor torques are shown in Figure 6.

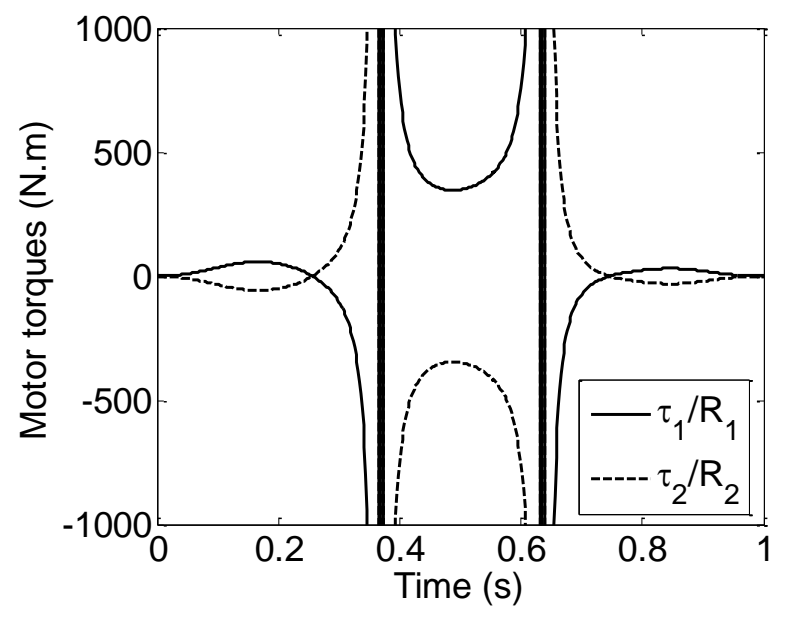

Figure 6

Motor torques required in Case 2

\subsection{Case 3: An Admissible Trajectory}

It is seen in Case 2 that when $t_{\mathrm{s}}$ is set to $0.5005 \mathrm{~s}$, there are two distinct real solutions for $a_{11}$. The consistent trajectory that is planned by choosing the first of them results in additional unintended occurrences of the same singular point. In this last case, this problem is resolved by choosing the second root, that is $a_{11}=1901.94295206846$, and planning another consistent trajectory. The other nonzero coefficients are accordingly calculated as follows: $a_{5}=1076.66244289026, a_{6}=-7075.25516651967, a_{7}=19556.3391892448$, $a_{8}=-28841.053949587, a_{9}=23842.7417351358, a_{10}=-10460.3772032326$. The so-obtained polynomial is plotted against time in Figure 7. The determinant of $\mathbf{A}^{\mathrm{u}}$ vanishes while its first-order time derivative is nonzero at the intended singularity time $t_{\mathrm{s}}=0.5005 \mathrm{~s}$. Moreover, there is no additional unintended occurrence of the singularity since statement (i) holds true with $b_{2}=20921.3725>0$ and $\Delta_{2}=-1.2931 \times 10^{7}<0$. As a result, the necessary motor torques are continuous and bounded (see Figure 8). 


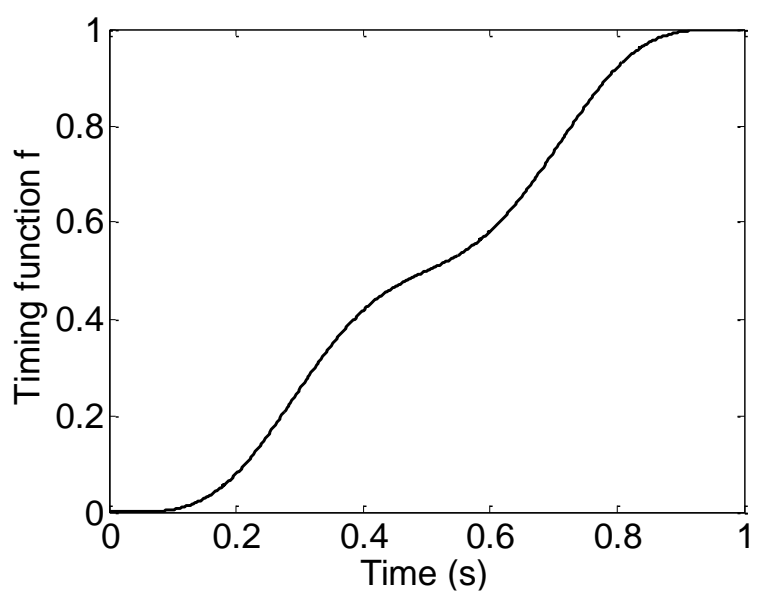

Figure 7

Timing function $f$ in Case 3

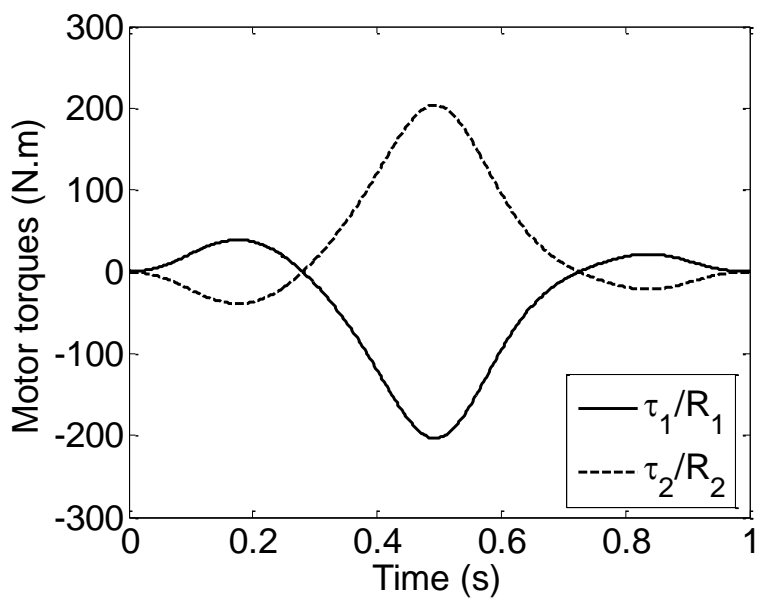

Figure 8

Motor torques required in Case 3

\section{Conclusions}

This paper contributes to the literature in the following ways:

- We develop a systematic step-by-step trajectory planning method for flexible-joint parallel robots passing through drive singular configurations.

- Our method generates a consistent trajectory with an eleventh-degree time polynomial, that is, with a time polynomial of a lower degree than that required in the related literature [19]. The importance of this 
achievement is that it enables transforming the problem of preventing unintentional reversal of the endpoint motion into the nonnegativity of a quadratic polynomial of time over the duration of the task.

- Although it is known that a trajectory, which is planned to be consistent at a drive singularity may result in undesired back-and-forth motion of the endpoint [11], we show here for the first time that this can lead to unintended multiple occurrences of that singularity, which should indeed be avoided.

- Four conditions are derived within the framework of the method to avoid undesired back-and-forth motion of the endpoint. Hence, the possibility of unintended multiple occurrences of the same singularity is prevented by the satisfaction of one of these four conditions.

- As previously shown in [9] for fully rigid parallel robots, we show in the present paper that the consistency requirement is also not sufficient for desingularization of flexible-joint parallel robots.

- The boundedness of the inverse dynamics solution is guaranteed by incorporating into the method a check for nonzeroness of the first time derivative of the vanishing determinant at the instant of singularity, in accordance with $[9,10]$.

In order to show the effectiveness of our approach, we also present a detailed case study considering the planar 5R parallel robot with joint flexibility. In Case 1, the occurrence of a high-order drive singularity is demonstrated for the first time for a flexible-joint parallel robot. Case 2 illustrates, again for the first time, undesirable additional occurrences of the same singularity. Finally, all these problems are overcome in Case 3 by using our developed trajectory planning method.

The practical importance of this study is that it provides an efficient way of enabling the utilization of the entire workspace of flexible-joint parallel robots. In applications, this joint flexibility can be due to inevitable structural elasticity and damping of drive transmission elements or can be intentionally introduced for avoiding damage to the motors in case of unexpected impact loads. The method developed in this study can be employed in all these possible situations.

\section{References}

[1] H. Simas, R. Simoni and D. Martins. Triflex II: Design and analysis of a self-aligning parallel mechanism with asymmetrical kinematic structure. Meccanica, 52(11-12):2991-3002, 2017

[2] J. Somló, G. D. Varga, M. Zenkl and B. Mikó. The „Phantom” Delta robot A new device for parallel robot investigations. Acta Polytechnica Hungarica, 15(4):143-160, 2018 
[3] J. Somló. General triangle parallel robot (GTPR) Basic features of a new robot type - kinematics and related application issues. Acta Polytechnica Hungarica, 16(5):7-24, 2019

[4] L.-T. Schreiber and C. Gosselin. Schönflies motion PARAllel robot (SPARA): A kinematically redundant parallel robot with unlimited rotation capabilities. IEEE/ASME Transactions on Mechatronics, 24(5):2273-2281, 2019

[5] C. Gosselin and J. Angeles. Singularity analysis of closed-loop kinematic chains. IEEE Transactions on Robotics and Automation, 6(3):281-290, 1990

[6] S. K. Ider. Inverse dynamics of parallel manipulators in the presence of drive singularities. Mechanism and Machine Theory, 40(1):33-44, 2005

[7] C. K. K. Jui and Q. Sun. Path tracking of parallel manipulators in the presence of force singularity. ASME Journal of Dynamic Systems, Measurement, and Control, 127(4):550-563, 2005

[8] S. Briot and V. Arakelian. Optimal force generation in parallel manipulators for passing through the singular positions. The International Journal of Robotics Research, 27(8):967-983, 2008

[9] M. Özdemir. Removal of singularities in the inverse dynamics of parallel robots. Mechanism and Machine Theory, 107:71-86, 2017

[10] M. Özdemir. High-order singularities of 5R planar parallel robots. Robotica, 37(2):233-245, 2019

[11] S. K. Ider. Singularity robust inverse dynamics of planar 2-RPR parallel manipulators. Proc. IMechE Part C: J. Mechanical Engineering Science, 218(7):721-730, 2004

[12] M. Özdemir. Optimization in motion planning for parallel robots passing through singular positions. Journal of the Faculty of Engineering and Architecture of Gazi University, 32(4):1089-1096, 2017

[13] M. W. Spong. Modeling and control of elastic joint robots. ASME Journal of Dynamic Systems, Measurement, and Control, 109(4):310-318, 1987

[14] M. W. Spong. Underactuated Mechanical Systems: In: Control Problems in Robotics and Automation, B. Siciliano and K. P. Valavanis (Eds.), Springer-Verlag London Limited, 1998, pp. 135-150. Series: Lecture Notes in Control and Information Sciences, Vol. 230, Editor: M. Thoma

[15] S. K. Ider and M. K. Özgören. Trajectory tracking control of flexible-joint robots. Computers \& Structures, 76(6):757-763, 2000

[16] S. K. Ider and O. Korkmaz. Trajectory tracking control of parallel robots in the presence of joint drive flexibility. Journal of Sound and Vibration, 319(1-2):77-90, 2009 
[17] S. Kilıçaslan. Tracking control of elastic joint parallel robots via statedependent Riccati equation. Turkish Journal of Electrical Engineering \& Computer Sciences, 23(2):522-538, 2015

[18] F. A. Lara-Molina, D. Dumur and K. A. Takano. Multi-objective optimal design of flexible-joint parallel robot. Engineering Computations, 35(8):2775-2801, 2018

[19] S. Briot and V. Arakelian. On the dynamic properties of rigid-link flexiblejoint parallel manipulators in the presence of Type 2 singularities. ASME Journal of Mechanisms and Robotics, 2(2):021004, 2010

[20] A. De Luca and W. Book. Robots with Flexible Elements. In: Springer Handbook of Robotics, B. Siciliano and O. Khatib (Eds.), Springer-Verlag Berlin Heidelberg, 2008, pp. 287-319

[21] M. J. Tobias. Matrices in Engineering Problems. In: Synthesis Lectures on Mathematics and Statistics, Series Editor: S. G. Krantz, Morgan \& Claypool Publishers, 2011 